\title{
The structural basis for regulated assembly and function of the transcriptional activator NtrC
}

\author{
Sacha De Carlo, ${ }^{1,7}$ Baoyu Chen, ${ }^{3,7}$ Timothy R. Hoover, ${ }^{5}$ Elena Kondrashkina, ${ }^{6}$ Eva Nogales, ${ }^{1,2,9}$ \\ and B. Tracy Nixon ${ }^{4,8}$ \\ ${ }^{1}$ Howard Hughes Medical Institute, Department of Molecular and Cell Biology, University of California at Berkeley, \\ Berkeley, California 94720, USA; ${ }^{2}$ Lawrence Berkeley National Laboratory, Berkeley, California 94720, USA; ${ }^{3}$ Integrative \\ Biosciences Graduate Degree Program-Chemical Biology and ${ }^{4}$ Department of Biochemistry and Molecular Biology, The \\ Pennsylvania State University, University Park, Pennsylvania 16802, USA; ${ }^{5}$ Department of Microbiology, University of \\ Georgia, Athens, Georgia 30602, USA; ${ }^{6}$ BioCAT at APS/Argonne National Lab, Illinois Institute of Technology, \\ Argonne, Illinois 60439, USA
}

In two-component signal transduction, an input triggers phosphorylation of receiver domains that regulate the status of output modules. One such module is the AAA+ ATPase domain in bacterial enhancer-binding proteins that remodel the $\sigma^{54}$ form of RNA polymerase. We report X-ray solution scattering and electron microscopy structures of the activated, full-length nitrogen-regulatory protein $\mathrm{C}(\mathrm{Ntr} \mathrm{C})$ showing a novel mechanism for regulation of AAA+ ATPase assembly via the juxtaposition of the receiver domains and ATPase ring. Accompanying the hydrolysis cycle that is required for transcriptional activation, we observed major order-disorder changes in the GAFTGA loops involved in $\sigma^{54}$ binding, as well as in the DNA-binding domains.

[Keywords: NtrC; gene regulation; AAA + ATPase assembly; transcription activation; enhancer-binding protein; two-component signal transduction]

Supplemental material is available at http://www.genesdev.org.

Received February 7, 2006; revised version accepted April 4, 2006.

The $\sigma^{54}$ holoenzyme form of RNA polymerase forms closed DNA complexes that open only with the help of enhancer-binding proteins (EBPs). For the nitrogen-regulatory protein $\mathrm{C}$ of enteric bacteria ( $\mathrm{NtrC}$ ), such activity starts a cascade of events that may ultimately lead to the activation of transcription for as much as $2 \%$ of the genome (Zimmer et al. 2000). Sequence analysis of $>1600 \sigma^{54}$-dependent transcriptional activators shows that in addition to their oligomerization/AAA+ ATPase domain, they also have a DNA-binding domain and a regulatory domain, which in $50 \%$ of cases is a twocomponent receiver domain (Bateman et al. 2004). The helix-turn-helix DNA-binding domain recognizes enhancer-like sequences between 100 and 150 bp upstream of the promoter. In their inactive states these proteins are usually dimers that bind to pairs of tandem sites in the enhancer elements. Upon activation via the regulatory domains, they oligomerize into ATPase-active rings

\footnotetext{
${ }^{7}$ These authors contributed equally to this work. Corresponding authors.

${ }^{8}$ E-MAIL btn1@psu.edu; FAX (814) 863-7024.

${ }^{9}$ E-MAIL enogales@lbl.gov; FAX (510) 642-8806.

Article is online at http://www.genesdev.org/cgi/doi/10.1101/gad.1418306.
}

that use the energy from ATP hydrolysis to physically remodel closed complexes of $\sigma^{54}$ holoenzyme and promoter DNA (Rombel et al. 1998; Neuwald et al. 1999; Chaney et al. 2001; Lee et al. 2003).

Crystal or NMR structures are available for several isolated domains and truncation constructs of EBPs including NtrC, DetD, PspF, ZraR, and NtrC1 (Volkman et al. 1995; Kern et al. 1999; Pelton et al. 1999; Meyer et al. 2001; Park et al. 2002; Hastings et al. 2003; Lee et al. 2003; Doucleff et al. 2005; Rappas et al. 2005; Sallai and Tucker 2005). Existing structural information on DctD and $\mathrm{NtrCl}$ has been used to provide a model of how two-component signal transduction can regulate assembly of their AAA+ ATPase domains (Lee et al. 2003), but this model fails to explain regulation in the closely related protein $\mathrm{NtrC}(40 \%$ sequence identity, $60 \%$ sequence similarity) (Lee 2000; Lee et al. 2000; Hastings et al. 2003). Here we report both SAXS/WAXS (small- and wide-angle X-ray scattering) and EM (electron microscopyl structures of the full-length, activated form of NtrC from Salmonella typhimurium. Docking of atomic models for the individual domains into the structures reveals their organization within the activated ring and uncovers a novel mechanism for the use of two-compo- 
De Carlo et al.

nent signal transduction to regulate the assembly of AAA + ATPase domains. Comparison of the ADP-versus $\mathrm{ADP}-\mathrm{AlF}_{\mathrm{X}}$-bound structures shows that in the latter the DNA-binding domain becomes more ordered with respect to other domains, and the GAFTGA loop region extends outward on the other side of the ring. These two features are of potential importance in the activation process.

\section{Results}

Our studies of the activated, oligomeric form of NtrC were facilitated by conditions that stably activate an NtrC variant that combined an S160F mutation, which favors an equilibrium toward the active, oligomeric state, and three alanine substitutions (R456A, N457A, and $\mathrm{R} 461 \mathrm{~A}$ ) in the DNA-binding domain that prevent aggregation of the protein in the activated state (Rombel et al. 1998). Initial sedimentation studies showed that upon activation by $\mathrm{Mg}^{2+} / \mathrm{BeF}_{3}{ }^{-}$the protein shifts from $\sim 4.5 \mathrm{~S}$ to $\sim 10 \mathrm{~S}$ particles in a fully reversible manner through a range of intermediate stoichiometries from dimer to hexamer (Supplementary Fig. S1).

\section{$X$-ray scattering model and domain organization}

Recent advances in the collection and analysis of SAXS/ WAXS (small- and wide-angle X-ray scattering) data have made it possible to model the low-resolution structure of samples in solution (Vachette et al. 2003; Koch 2005). We collected SAXS/WAXS data for the activated, oligomeric NtrC bound to ADP. Analysis of the data was facilitated by the known hexameric nature of the complex obtained from electron microscopy (see Supplementary Fig. S1). The average structure produced from the scattering data is shown in Figure 1A. Assignment of the different domains within the density (shown by different colors in Fig. 1A) started by assuming a central ring of the AAA+ ATPase (Fig. 1A, white). After docking into the central portion of the structure a hexameric model of the AAA+ ATPase (see below), it became obvious that the receiver domains (Fig. 1A, orange) would have to occupy the peripheral lobes to satisfy previously reported biochemical and NMR data that placed helix 4 of a receiver domain close to helix 1 of an ATPase domain (Fig. 1C; Lee et al. 2000; Hastings et al. 2003). Finally, the DNA-binding domains had to occupy the remaining volume below the ATPase ring surrounding the central pore on the "bottom" side (Fig. 1A, blue). A full model of the activated, full-length NtrC was built by manually docking the available high-resolution structures of each domain as summarized above (Fig. 1B-E). Overall, there is an excellent size correspondence between the atomic models of the different domains and their assigned density regions in the structure. The sixfold symmetric ATPase ring is based on the NtrCl crystal structure (Lee et al. 2003; see Materials and Methods) after changing the symmetry from seven to six. Figure 1, B-D, shows consecutive ATPase domains in the ring in alternating
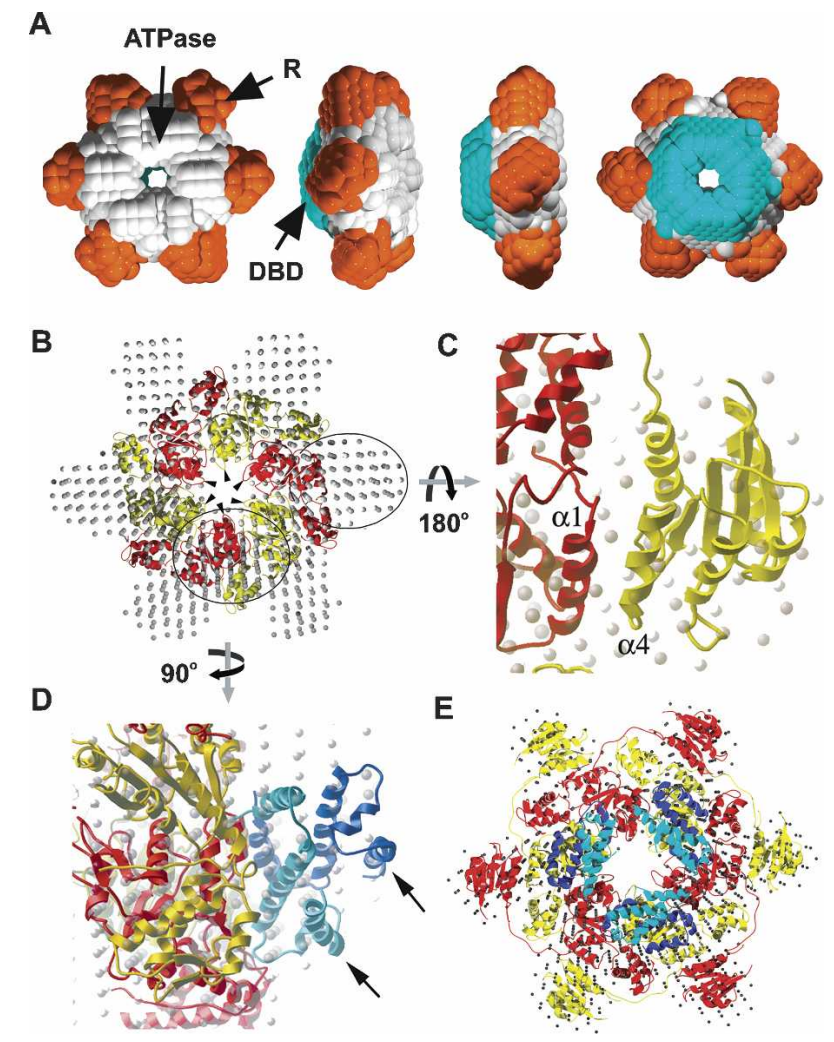

Figure 1. SAXS/WAXS averaged structure and pseudoatomic model for the activated full-length NtrC. $(A)$ The structure shows the juxtaposition of receiver (R, orange), ATPase (white), and DNA-binding (DBD, cyan) domains, identified by superimposing a hexameric ATPase model and requiring close proximity between helix $\alpha 4$ of the receiver domains and helix $\alpha 1$ of the ATPase domains. $(B)$ A hexameric ATPase ring (alternating red and yellow) was fitted within the SAXS/WAXS solution structure (spheres denoting scattering centers; small arrowheads pointing to GAFTGA loops by the central hole). Enlargements of the circled regions illustrate placement of $(C)$ a receiver domain (yellow), keeping helix $\alpha 4$ (residues $86-95$ ) close to helix $\alpha 1$ (residues 144-157) of the ATPase domain (red) (see Supplementary Fig. S6A for details); and $(D)$ a dimer of the DNAbinding domains (cyan and blue), keeping the recognition helices surface-exposed (arrows). (E) Final model after placing all six receiver and DNA-binding domains, using sixfold and threefold symmetry, respectively, and linking the receiver and ATPase domains with a 25-residue coil. The DNA-binding domains face the viewer.

yellow and red colors in order to help visualize the individual subunits. The same color scheme was used in the rest of the figures in this paper. The NMR structure of the activated NtrC receiver domain (Hastings et al. 2003) was then positioned on the outer knobs, and the orientation was set to accommodate existing NMR chemical shift data (Hastings et al. 2003), Fe-BABE cleavage data, and the phenotypes of mutations associated with the cleavage studies (Lee 2000; Lee et al. 2000; S. Kustu, pers. comm.). These data demand a close proximity between helix 4 of the receiver domain and helix 1 of the ATPase domain, and suggest that essential roles are played by the 
surface-exposed residues L87, A90, and V91 of helix 4, and residues G142, R152, and R156 of the ATPase domain. Keeping the helices close to each other while maximizing the fit with the SAXS/WAXS model gave two possible orientations rotated roughly $90^{\circ}$ relative to one another. The orientation shown in Figure 1C is favored because it could be fine-tuned to include several reasonable contacts that are consistent with the observed phenotypes (see fitting into EM structures, below) (Supplementary Fig. S6). Finally, three copies of the NMR structure of the DNA-binding domain dimer (Pelton et al. 1999) were used to fill the unoccupied portion of the structure located below the ring, maximizing contact with the ATPase domains while keeping the DNAbinding helix on the outer surface of the model (Fig. $1 \mathrm{D}, \mathrm{E})$. Notice that because we had imposed sixfold symmetry to generate the solution model, the SAXS/WAXS structure does not match the $3 \times 2$-fold symmetric DNAbinding domains (but see below).

\section{D EM reconstruction and nucleotide effects}

The full-length, beryllofluoride-activated protein was also used to generate electron microscopy (EM) 3D reconstructions from negatively stained samples. The structures of the ring in its ADP and ADP-AlF ${ }_{x}$ forms are shown in Figure 2, A and B, respectively. The reconstruction of the ADP-bound protein is fairly flat, with a diameter of $\sim 180 \AA$, and a central pore of $\sim 34 \AA$ in diameter. The central region is surrounded by six distinct densities in a nearly perfect hexameric conformation (symmetry not imposed) (see Supplementary Fig. S5). The ADP-AlF ${ }_{x}$ reconstruction is clearly thicker, showing extra density both on the top (arrow) and bottom (arrowhead) sides of the main central domain (Fig. 2C, left). Additional, finer differences are also seen, including a small inward movement and clockwise rotation of the peripheral receiver domains for the $\mathrm{ADP}-\mathrm{AlF}_{\mathrm{x}}$ structure with respect to that bound to ADP (Fig. 2C). There is also a slight decrease in the size of the central pore in this ADP-AlF $F_{x}$ conformation (30 ̊ for the ADP-AlF $F_{x}$ state).

The docking model shown in Figure 1E was manually fitted into the EM density maps for both the ADP-AlF and ADP bound protein (Fig. 3A-C, respectively). The receiver domains had to be slightly adjusted to optimally fit into the EM maps. Importantly, the GAFTGA-loop regions of the ATPase domain are only fitted within the ADP-AlF $F_{x}$ structure (Fig. 3, cf. B and C), which is also the only one of the two that can accommodate the DNAbinding domains (Fig. 3, cf. B and C; DNA-binding domains shown in blue for the ADP-AlF ${ }_{x}$ structure). The EM density for these domains is distributed in three distinct lobes, each one of them fitting the mass of the NMR structure of a dimer. Thus, there is a symmetry mismatch between the central ATPase domains (and the interacting receiver domains in the periphery), which are arranged front-to-back in a hexamer, and the DNA-binding domains, organized into three back-to-back dimers. It is important to mention here that the three domains within each subunit are connected by long, flexible link-
A
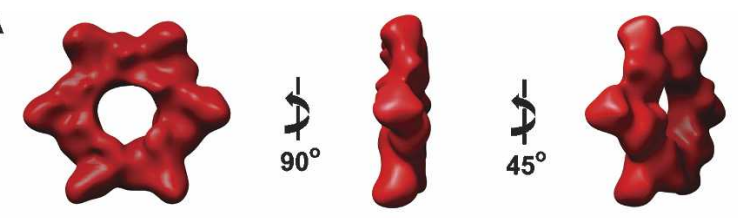

B
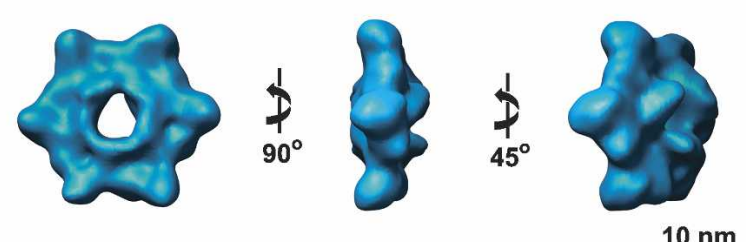

C
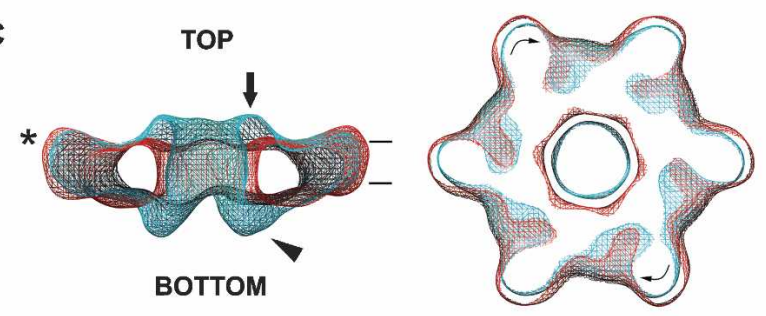

ADP

ADP-AIF $F_{X}$

$6 \mathrm{~nm}$

Figure 2. EM model for activated NtrC. (A) $3 \mathrm{D}$ reconstruction of the ADP-bound structure (red) at $25 \AA$ resolution: top view (left), side view (center), and slanted view (right). (B) Reconstruction of the ADP-AlF ${ }_{x}$-bound structure (cyan) at $28 \AA$ resolution. (C) Superposition of the two EM maps. The ADP-bound state (red mesh) and the ADP-AlF ${ }_{x}$ state (cyan mesh) are rendered with the same $4.6-\sigma$ threshold representing $~ 100 \%$ of the expected molecular volume. The slab through the side view on the left shows clear extra densities in the ADP-AlF - -bound structure; the arrow on the top indicates additional mass in the ADP$\mathrm{AlF}_{X}$ structure where the GAFTGA loops are located; the arrowhead on the bottom indicates extra mass corresponding to the presence of the DNA-binding domains (see also Fig. 3). The right panel shows a slab through the front view (the thickness is indicated in the panel on the left to illustrate a detectable inward movement and slight $\mathrm{CW}$ rotation of the receiver domains in the ADP- $\mathrm{AlF}_{\mathrm{x}}$ state (curved arrows marked by an asterisk on the left panel).

ers that allow for this kind of arrangement. Interestingly, the DNA-binding domains are only visible in the nucleotide transition state, while their disappearance in the ADP-bound form indicates that they are disordered after phosphate release. The difference between the structures derived for the ADP-bound form of the protein using $\mathrm{X}$-ray scattering (where density is visible for the DNAbinding domains) and EM (where density is lacking) arises from the nature of the experiments. Conformationally flexible regions contribute to the SAXS/WAXS structure because the scattering signals are added incoherently, while coherent addition of images containing information about both phase and amplitude causes such flexible regions to be averaged out in the EM reconstruction.

In summary, the model in Figure 3 confirms a central ATPase domain, with six receiver domains on the outer 

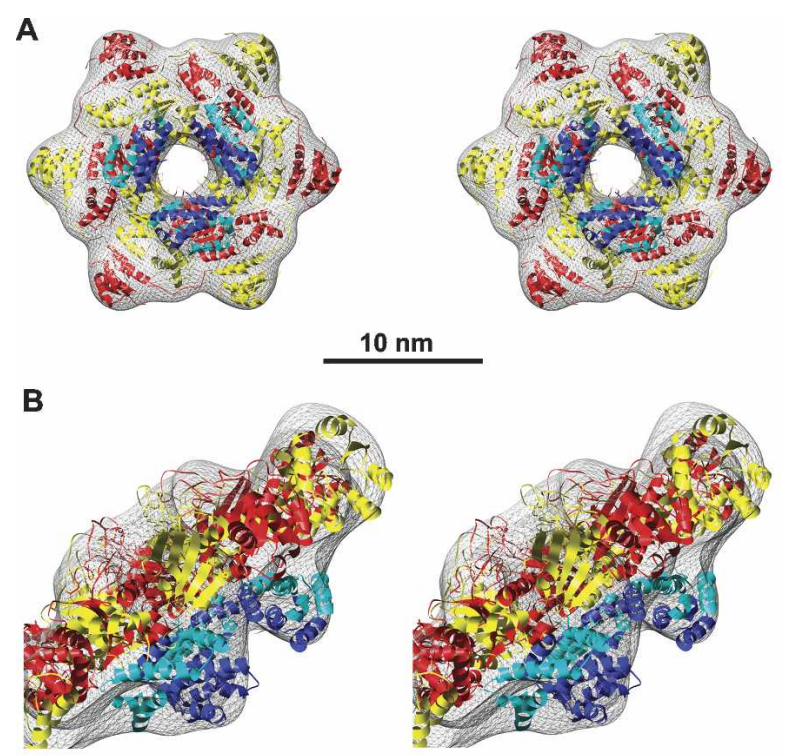

ADP-AIF $_{\mathbf{X}}$

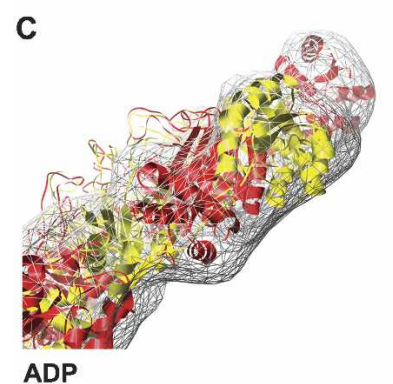

ADP

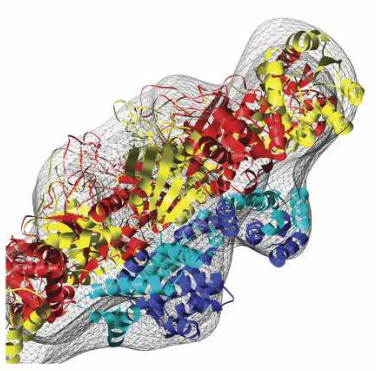

$5 \mathrm{~nm}$

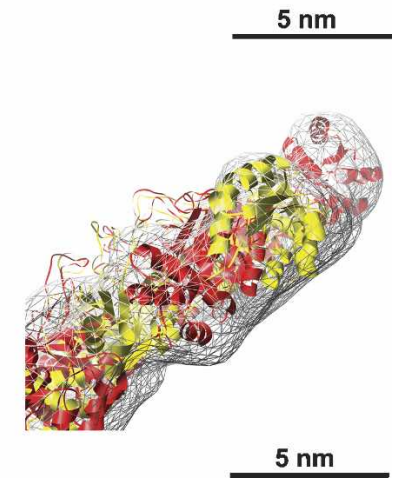

Figure 3. Full-length model of NtrC for the ADP and ADP$\mathrm{AlF}_{x}$ states. (A) Stereo top view of the ADP-AlF ${ }_{x}$ pseudoatomic model created by manual fitting within the gray mesh of the EM density of the model first presented in Figure 1E after additional adjustments. The color code is the same as in Figure 1E with the central and the receiver domains in alternating red/yellow, and the DNA-binding domain dimers in blue/cyan. The DNA-binding domains face the viewer. (B) Stereo side view of the ADP$\mathrm{AlF}_{x}$ structure. The density above the ATPase domain accommodates well the GAFTGA loop regions. $(C)$ The same view as in $B$ but for the ADP state. There is not enough density to account for the DNA-binding domains (omitted for emphasis) or the GAFTGA loops.

radius tightly packed against it, and three dimers of DNA-binding domains underneath the main ring that become flexible and likely detach from the central ring upon release of inorganic phosphate.

\section{Discussion}

Mechanism of positive activation in $\sigma^{54}$-dependent $A A A+$ ATPases: direct contribution to hexamer stability by phosphorylated $\mathrm{NtrC}$ receiver domains

Regulation of the assembly of $\sigma^{54}$-dependent AAA+ ATPases can be achieved by either a negative or a positive mechanism. In negative regulation, typified by
NtrC1 and DctD, the unphosphorylated receiver domain forms a homodimer that, through an extensive interface with an ATPase dimer, represses spontaneous assembly of the intrinsically competent ATPase domain to its active ring form. Phosphorylation stabilizes an alternative homodimer configuration that relieves the repressed state, permitting the ATPase to assemble (Fig. 4A, top; Park et al. 2002; Lee et al. 2003). In agreement with this

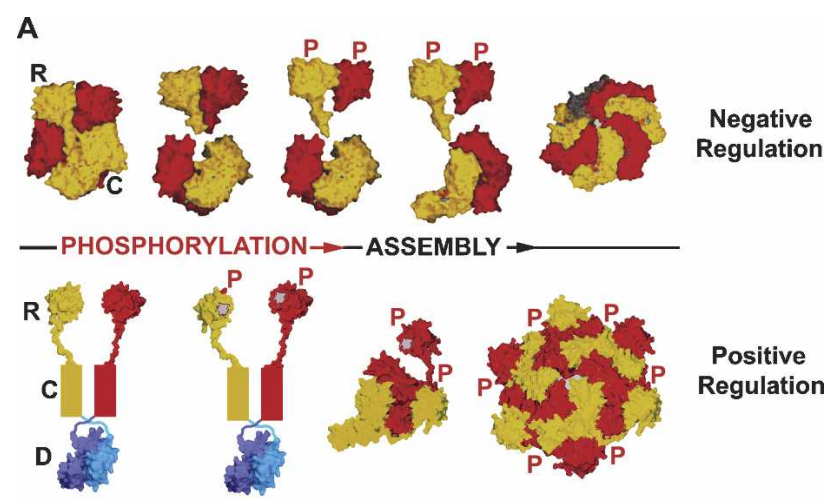

B

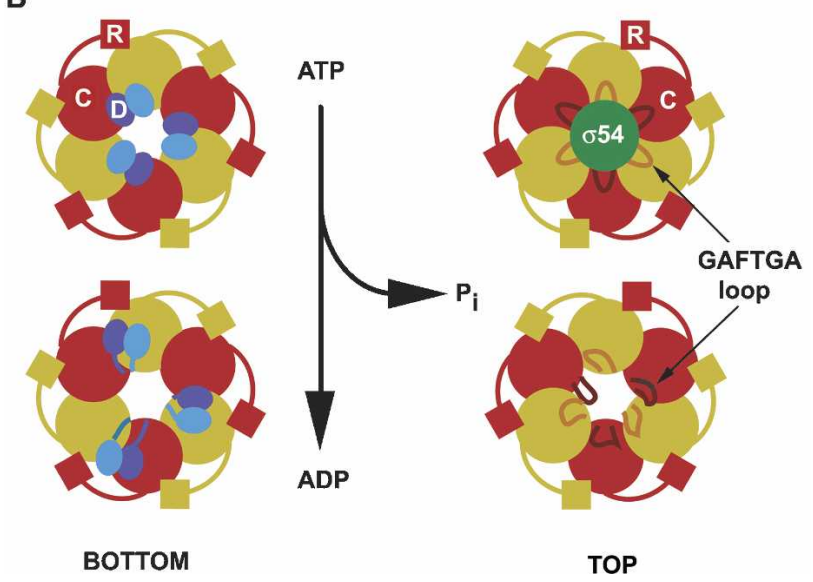

Figure 4. Mechanism of oligomerization and activation. $(A)$ Comparison of the negative mechanism for regulating assembly of DctD and NtrC1 (top) with the positive regulation in NtrC (bottom). In the negative mechanism, phosphorylation (at site labeled P) stabilizes an alternate dimer conformation of receiver domains $(\mathrm{R})$ that fails to inhibit spontaneous ring assembly by the ATPase domains (C). In the positive mechanism, phosphorylation exposes a hydrophobic patch (pink) to mediate alternating interactions between yellow and red subunits that facilitates ring assembly (DNA-binding domains $[\mathrm{D}$, blue and cyan] are hidden under the ring). (B) Schematic model for the mechanism of $\sigma^{54}$ remodeling by NtrC. (R) Receiver domain, (C) ATPase domain, (D) DNA-binding domain. In the ADP-AlF $F_{x}$ form, corresponding to the transition state for hydrolysis (top left and right), the DNA-binding domains are closely packed against the ATPase ring (left), likely distorting the enhancer DNA in the process, while the exposed and ordered GAFTGA region is able to interact with $\sigma^{54}$ (filled green circle) on the other side of the ring (top right). By the time $\mathrm{P}_{\mathrm{i}}$ has been released (bottom), the GAFTGA region has let go of $\sigma^{54}$ and become disordered (bottom right) and the DNA-binding regions detached from the central ring (bottom left), releasing their constraint on the DNA. 
mechanism, deletions that truncate or remove the receiver domain result in constitutively active proteins (see Doucleff et al. 2005). In stark contrast, positively regulated activators like $\mathrm{NtrC}$ require the presence of the phosphorylated receiver domain to function in ATPase and transcription activation assays (Drummond et al. 1990; Weiss et al. 1992a,b; Klose et al. 1993). Our structures, together with previous biochemical data showing that the activated receiver domain of one subunit is in contact with the ATPase domain of a second, nonidentical subunit, offer an explanation for the positive mode of regulation in NtrC. Each activated receiver domain binds to a peripheral edge of an adjacent subunit's ATPase domain. This pattern of intersubunit contacts persists around the outer edge of the ring, contributing directly to the hexamer's stability (Fig. 4A,B). Furthermore, it is likely that the phosphorylation-dependent assembly proceeds through the stabilization of back-tofront dimers of the ATPase domain, with similar juxtaposition between the receiver and ATPase domains as those seen in our hexamer. This is consistent with previous Fe-BABE cleavage studies (Lee 2000; Lee et al. 2000; S. Kustu, pers. comm.). These back-to-front dimers would then readily merge and close to form the final hexamer ring illustrated in Figure 4A. The hypothesis of back-to-front dimers as intermediates in the activation process is consistent with the multiple species of intermediate sedimentation velocities that we observed for partially activated NtrC (S160F, 3-Ala) (Supplementary Fig. S1).

The first helix of the ATPase domain, called $\alpha \mathrm{N}$, was suggested to propagate signals between the receiver domain and the rest of the ATPase (Osuna et al. 1997). Cysteine substitutions in the residues of helix $\alpha \mathrm{N}$, which were used in cleavage studies, resulted in a variety of phenotypes (Lee 2000; Lee et al. 2000). These include failure to activate, impaired DNA binding, and constitutive activation of NtrC. The model for activated NtrC presented here shows residues D149, F151, R152, and $\mathrm{R} 156$ of helix $\alpha \mathrm{N}$ facing helix 4 of the receiver domain, directly contacting residues L87, D88, A90, and V91 (see Supplementary Fig. S6). Mutations in this portion of helix $\alpha \mathrm{N}$ may thus exert their effects by enhancing or disrupting contact with the receiver domain rather than by affecting the remainder of the central domain.

DNA-binding, nucleotide cycle, and interaction with $\sigma^{54}$ : the transition state of NtrC exposes the GAFTGA loops and fixes the orientation of the DNA-binding domains

The complex operon glnAntrBC, encoding glutamine synthetase as well as the histidine kinase NtrB and NtrC itself, is one of the well-characterized genetic loci that are regulated by NtrC (for reviews, see Merrick and Edwards 1995; Reitzer 2003). At this locus, NtrC binds to an enhancer/operator region located between 100 and 150 bp upstream of $g \ln A$, and to an operator located between $g \ln A$ and $n \operatorname{tr} B$. Given that the $g \ln A$ enhancer region contains two tandem motifs for binding NtrC dimers, formation of a hexamer upon activation would imply that a third dimer would have to be recruited and incorporated to close the ring, possibly aided by binding to nonspecific DNA. Additional, low-affinity binding sites on the promoter-proximal side of the $g \ln A$ enhancer were seen in both in vitro (Hirschman et al. 1985) and then in in vivo footprints of activated NtrC (SasseDwight and Gralla 1988) and reported to be necessary for high-level transcription activation (Lilja et al. 2004). The DNA-binding domains are localized to the bottom side of the NtrC ring, and therefore binding to the enhancer is on the opposite face of the ATPase from its interaction site with $\sigma^{54}$ polymerase and promoter DNA, which occurs via the GAFTGA loop region (Lee et al. 2003). It seems that contact with enhancer DNA adds efficiency to formation of the NtrC oligomer (Porter et al. 1995), but is not essential for the remodeling of $\sigma^{54}$. At high concentrations many of the $\sigma^{54}$-dependent ATPase domains (including NtrC's) can act in the absence of DNAbinding activity and in an enhancer-independent fashion (North and Kustu 1997). However, substitutions in the GAFTGA loop of NtrC caused binding to the enhancer or even nonspecific DNA to act as a negative allosteric regulator of the interaction with RNA polymerase (Yan and Kustu 1999), and prior studies of DctD, activated by deleting its $\mathrm{N}$-terminal receiver domain, showed that ADP-AlF ${ }_{x \prime}$ but not ADP or ADP-BeF $F_{x}$ caused hypersensitivity to DNase I to appear between the tandem binding sites in the $\operatorname{dct} A$ UAS (Wang et al. 2003). These effects may be related to the change in relative flexibility in the connection of the DNA-binding domains with the ATPase ring that we observed during the hydrolysis cycle, which would have a dramatic effect on the structure of the DNA. As the DNA-binding domains clamp onto the ring in the ADP- $\mathrm{AlF}_{x}$ state, the DNA will be forced to bend significantly between the tandem activator binding sites in order to bring them together, while this constraint would be relaxed after phosphate release when the DNA-binding domains lose their tight association with the ring (Fig. 3, cf. B and C). Such conformational dynamics may help NtrC assemble into a full hexamer or increase the ability of its ATPase domain to interact with the $\sigma$ factor at the other side of the ring (Fig. 4B). The subsidiary, low-affinity sites on the promoter proximal side of the $g \ln A$ enhancer may contribute to these dynamics (Lilja et al. 2004).

The current model for interaction of a bacterial enhancer-binding protein with $\sigma^{54}$ rests upon the ADP$\mathrm{AlF}_{x}$ transition state stabilizing a tight contact between them (Chaney et al. 2001). An EM structure for such a complex was recently reported for $\mathrm{PspF} / \sigma^{54}$, in which two-thirds of the mass for $\sigma^{54}$ was bound to the top side of the ATPase, slightly offset from its center (Rappas et al. 2005). Although only observed at very low threshold, density for the GAFTGA loops appeared to reach up from the edge of the inner pore to contact the $\sigma$ factor, which, contoured at a more typical level, seemed to float above the ring. Our structures of ADP- and ADP-AlF $F_{x}$ bound NtrC (S160F, 3-Ala) provide data supporting that model, as well as clear evidence that the GAFTGA loop region 
forms a raised rim above the pore of the ring in the ADP$\mathrm{AlF}_{\mathrm{X}}$ state, but then relaxes in the ADP-bound state (Figs. 3 [cf. B and C], 4B).

\section{Conclusions}

The structural model that we describe for activated $\mathrm{NtrC}$, in which the activated receiver domain of one subunit is in contact with the ATPase domain of a second one to stabilize ring assembly, differs dramatically from the model proposed to explain how two-component signal transduction regulates assembly of AAA + ATPase domains in the enhancer-binding proteins $\mathrm{NtrCl}$ and DctD (Lee et al. 2003; Doucleff et al. 2005). Consequently, the new model establishes the structural differences underlying positive versus negative regulation for this family of enhancer-binding proteins. Our structure of the activated NtrC suggests that phenotypes of substitutions in residues 149-156 of NtrC helix $\alpha \mathrm{N}$ arise from specific changes in the interface between ATPase and receiver domains, and it suggests intermediates for the assembly process. Finally, we show that order-disorder transitions in the GAFTGA loop region and DNAbinding domains accompany the nucleotide hydrolysis cycle. Since coupling of hydrolysis, DNA binding, and $\sigma$-factor remodeling can be dramatically perturbed (Yan and Kustu 1999), these transitions must be understood to learn how these AAA+ ATPases perform mechanical work to remodel the $\sigma^{54}$ form of RNA polymerase.

\section{Materials and methods}

\section{Protein purification}

NtrC (S160F, 3-Ala) was expressed in a 20-L fermentation using a modified broth containing yeast extract $(40 \mathrm{~g} / \mathrm{L})$, Tryptone $(20$ $\mathrm{g} / \mathrm{L})$, glycerol $(30 \mathrm{~g} / \mathrm{L}), \mathrm{NaH}_{2} \mathrm{PO}_{4}{ }^{\star} \mathrm{H}_{2} \mathrm{O}(8 \mathrm{~g} / \mathrm{L}), \mathrm{K}_{2} \mathrm{HPO}_{4}(7 \mathrm{~g} / \mathrm{L})$, and antifoam polypropylene glycol $2000(0.5 \mathrm{mg} / \mathrm{L})$. Cell paste $(15 \mathrm{~g})$ that had been resuspended in $25 \mathrm{mM}$ sodium phosphate buffer ( $\mathrm{pH}$ 7.9) containing $500 \mathrm{mM} \mathrm{KCl}, 5 \%(\mathrm{w} / \mathrm{v})$ glycerol, and 1 tablet of Complete Protease Inhibitor (EDTA-free) was sonicated and the resulting extract cleared by centrifugation at $100,000 \mathrm{~g}$ for $45 \mathrm{~min}$. Although the protein was not His-tagged, it was found to stick to a 4-mL Ni-NTA column if loaded at 1 $\mathrm{mL} / \mathrm{min}$ and washed at $4 \mathrm{~mL} / \mathrm{min}$. Bound protein was eluted with a linear gradient of the same buffer supplemented with 400 $\mathrm{mM}$ imidazole. Eluted sample was immediately desalted into buffer containing $10 \mathrm{mM}$ Tris- $\mathrm{HCl}(\mathrm{pH} 7.9), 50 \mathrm{mM} \mathrm{KCl}, 5 \%$ (w/v) glycerol, $200 \mu \mathrm{M} \mathrm{BeCl}_{2}$ (added from a $1 \mathrm{M}$ stock solution), $5 \mathrm{mM} \mathrm{NaF}$ (added from a $2.5 \mathrm{M}$ stock slurry), and $5 \mathrm{mM} \mathrm{MgCl}_{2}$, or the same buffer minus $\mathrm{BeCl}_{2}$ and $\mathrm{NaF}$ for the apo protein. The protein solutions were then applied to a Q-Sepharose column and eluted with a salt gradient (50 $\mathrm{mM}$ to $1 \mathrm{M} \mathrm{KCl}$ ) at $\sim 250 \mathrm{mM}$ concentration. Samples, at $\sim 4 \mathrm{mg} / \mathrm{mL}$, were brought to $1 \mathrm{mM}$ TCEP and $30 \%$ glycerol $(\mathrm{v} / \mathrm{v})$ and stored in $12-\mathrm{mg}$ aliquots at $-60^{\circ} \mathrm{C}$ prior to a final gel filtration purification step, the exact conditions for which varied depending on the intended use. The purity of proteins was assessed by SDS-PAGE (Supplementary Fig. S1A).

\section{Analytical ultracentrifugation}

$\mathrm{NtrC}$ (S160F, 3-Ala) protein was isolated from a size exclusion column using buffer with or without $\mathrm{Mg}^{2+} / \mathrm{BeF}_{3}{ }^{-}\left(5 \mathrm{mM} \mathrm{MgCl}_{2}\right.$,
$200 \mu \mathrm{M} \mathrm{BeCl}_{2}$, and $5 \mathrm{mM} \mathrm{NaF}$ ) and diluted to a concentration of $0.5 \mathrm{mg} / \mathrm{mL}$. EDTA $(5 \mathrm{mM})$ was added to one sample containing $\mathrm{Mg}^{2+} / \mathrm{BeF}_{3}{ }^{-}$, and after overnight incubation they were spun at $48,000 \mathrm{rpm}$ in an aluminum centerpiece in a Beckman XL-I ultracentrifuge equilibrated to $20^{\circ} \mathrm{C}$. After completion, the sample with $5 \mathrm{mM}$ EDTA was recovered, additional EDTA was added to yield $10 \mathrm{mM}$ final concentration, and the sample was resedimented. Optical density was measured, and sedimentation data were processed and analyzed using the enhanced van Holde-Weischet method (Hansen et al. 1994) of Ultrascan II (Demeler 2005). For equilibrium experiments, nine samples in buffer containing $\mathrm{Mg}^{2+} / \mathrm{BeF}_{3}{ }^{-}$were prepared by 0.3 -fold dilutions of $1 \mathrm{mg} / \mathrm{mL}$ solutions and equilibrated at speeds 9500 , 12,200 , and 16,500 $\mathrm{rpm}(1.5,2.5$, and $4.5 \sigma$ for hexamer) before radial absorption scans were obtained. Various equilibrium models were fitted to the data using WinNonlin (distributed by Jeffrey W. Lary and the National Analytical Ultracentrifugation Facility of the University of Connecticut Biotechnology Center). For NtrC (S160F, 3-Ala), vbar and MolWt $(0.7409 \mathrm{~mL} / \mathrm{g}$ and $52,118 \mathrm{~g} / \mathrm{mol}$ of monomer, respectively) were calculated from amino acid sequence using SEDNTERP /distributed by John Philo via the Reversible Associations in Structural and Molecular Biology software depot).

In the presence of $5 \mathrm{mM} \mathrm{MgCl}$, the protein sedimented as a broad population of $\sim 5 \mathrm{~S}$ particles with clear evidence of heterogeneity near $5 \mathrm{~S}$ and a small amount of larger species up to $\sim 10 \mathrm{~S}$ size (Supplementary Fig. S1B,C, solid black lines). The heterogeneity is presumably due to the S160F substitution, which has been shown to weakly activate NtrC (Popham et al. 1989; Dixon et al. 1991; Weiss et al. 1991). When $200 \mu \mathrm{M} \mathrm{BeCl}_{2}$ and $5 \mathrm{mM} \mathrm{NaF}$ were included in the buffer to fully activate the protein (Yan et al. 1999) the population shifted to an $\sim 10 \mathrm{~S}$ particle size, with evidence of heterogeneity between $9.5 \mathrm{~S}$ and $10 \mathrm{~S}$ (Supplementary Fig. S1B,C, blue lines). Under these activating conditions, equilibrium sedimentation at four speeds over a 32-fold concentration range gave radial distribution profiles that were most consistent with a hexamer single species model (Supplementary Fig. S1D). Consistently, electron microscopy (EM) images of negatively stained samples of the fully activated material showed hexamer rings that were further stabilized by the addition of ADP (Supplementary Fig. S1E) or

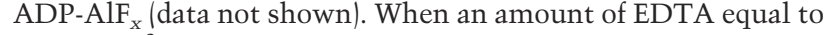
that of $\mathrm{Mg}^{2+}$ ion was added to the activation buffer, a mixture of $\sim 6 \mathrm{~S}$ to $\sim 10 \mathrm{~S}$ particles was observed (Supplementary Fig. S1B,C, magenta lines). Addition of twofold excess EDTA reduced the larger particle forms so that only $\sim 4.5$-S ones remained (Supplementary Fig. S1B,C, orange lines). These results show that upon activation, the protein shifts from $\sim 4.5 \mathrm{~S}$ to $\sim 10 \mathrm{~S}$ particle size in a fully reversible manner that transitions through a range of intermediate particle stoichiometries from dimer to hexamer. This result differs from prior suggestions that activated NtrC is an octamer (Rippe et al. 1998).

\section{Small- and wide-angle X-ray scattering (SAXS/WAXS)}

Experiments were conducted on the Biophysics Collaborative Access Team (BioCAT) undulator beamline 18-ID at the Advanced Photon Source, Argonne National Lab (Fischetti et al. 2004). An aliquot of NtrC (S160F, 3-Ala) containing $12 \mathrm{mg}$ of protein was thawed and diluted 1:6 with buffer containing 20 $\mathrm{mM}$ Tris (pH 7.9), $200 \mathrm{mM} \mathrm{KCl}, 200 \mu \mathrm{M} \mathrm{BeCl}_{2}, 5 \mathrm{mM} \mathrm{NaF}$, and $5 \mathrm{mM} \mathrm{MgCl}_{2}$ (thus making a final solution at $5 \%$ glycerol). The sample was then concentrated at $22^{\circ} \mathrm{C}$ to $\sim 0.5 \mathrm{~mL}(\sim 25-30 \mathrm{mg} /$ $\mathrm{mL}$ ) by centrifugation at $3500 \mathrm{rpm}$ in Amicon Ultra filters (10,000 MW cutoff). Centrifugation was interrupted every 10 min or less to remix the solution to minimize precipitation at 
the bottom of the filter and, in some instances, when $1 \mathrm{~mL}$ was reached, ADP was added from $100 \mathrm{mM}$ stock solution (pH 7.0) to a final concentration of $1 \mathrm{mM}$. The final sample $(0.5 \mathrm{~mL})$ was centrifuged at $10,000 \mathrm{~g}$ for $5 \mathrm{~min}$ and filtered through a $0.1 \mu \mathrm{m}$ Anotop filter.

In order to eliminate all aggregates, the final gel-filtration purification step was performed in-line with the exposure capillary, with scattering profiles (Supplementary Fig. S2A) and optical density at $280 \mathrm{~nm}$ (Supplementary Fig. S2B) being collected during the entire elution period. The filtered sample $(120 \mu \mathrm{L})$ was applied to a $24-\mathrm{mL}$ Superdex 200 gel filtration column. The column, pre-equilibrated with two volumes of the buffer, was eluted at $0.5 \mathrm{~mL} / \mathrm{min}$. The efflux was directed to the exposure capillary, $1.5 \mathrm{~mm}$ quartz, where samples were exposed to focused X-rays (12 keV and $2 \times 10^{13}$ photons/sec flux) for an average of $0.58 \pm 0.08 \mathrm{sec}$. Two-dimensional scattering patterns were obtained about every $15 \mathrm{sec}$ by using a $5 \times 9$-cm chargecoupled device (CCD) detector (Phillips et al. 2002) at a specimen-to-detector distance of $2.78 \mathrm{~m}$ (SAXS) or $0.24 \mathrm{~m}$ (WAXS). Radiation damage was minimized by including $5 \mathrm{mM}$ DTT in the sample solutions and by pumping them through the capillary at $8.5 \mu \mathrm{L} / \mathrm{sec}$ (Fischetti et al. 2003). Scattering intensity profiles over the Q range from 0.007 to $1.400 \AA^{-1}$ were calculated from radial averaging of the $2 \mathrm{D}$ scattering patterns using macros written by the APS staff for IGOR Pro (WaveMetrics, Inc.). Scattering profiles from protein plus buffer and from buffer alone (the latter taken before and after injected sample eluted from the column) were scaled using incident flux values integrated over the exposure time. Protein scattering profiles were then obtained by subtracting the averaged buffer profile from each profile of buffer plus protein. The PRIMUS program (Konarev et al. 2003) was used to merge SAXS and WAXS scattering data and to calculate the radius of gyration $(R g$, see Equation 1) from the slope of plots of $\ln (I)$ versus $Q^{2}$ as defined by the Guinier approximation (Equation 2).

$$
\begin{aligned}
R g & =\sqrt{\sum_{i} m_{i} r_{i}^{2} / \sum_{i} m_{i}} \\
\ln (I) & =\frac{R g^{2}}{3} Q^{2}+\ln \left(I_{0}\right)
\end{aligned}
$$

Guinier plots for these data were linear, demonstrating the absence of aggregates (e.g., the plot for the peak of NtrC [S160F, 3 -Ala] is shown in Supplementary Fig. S2C). $R g$ and $I_{0}$ values were thus calculated from the slopes and extrapolated intercepts of Guinier plots and graphed versus the elution volume for the main peak (Supplementary Fig. S2B). The $I_{0}$ values tracked the $\mathrm{OD}_{280 \mathrm{~nm}}$ profile, and the $R g$ value was seen to slowly decrease from 56 to $54 \AA$. Wide-angle scattering data were then collected in like fashion, for which plots of $\mathrm{OD}_{280 \mathrm{~nm}}$ and intensity at a $Q$-value of $0.1 \AA^{-1}$ versus elution volume showed the expected elution profile (Supplementary Fig. S2D).

Small- and wide-angle X-ray scattering data sets were scaled, merged, and cropped to generate the SAXS/WAXS scattering profile shown in Supplementary Figure S3A. The combined data span the Q-range of $0.004-1.25 \AA^{-1}$. Both Moore's autocorrelation function (Moore 1980) and the indirect transform of GNOM (Svergun 1992) were used to survey different values for the maximum dimension of the particles, $D_{\max }$ (Supplementary Fig. S3B). As expected for a monodisperse sample, the interatomic distance distribution function $[p(R)]$ approached zero as larger $D_{\max }$ values were sampled. The $R g$ values for each $D_{\max }$ surveyed stabilized at $54.1 \pm 0.2 \AA$ (Supplementary Fig. S3B), independently confirming the estimate from the Guinier plots presented above. The reduced $\chi^{2}$ of the autocorrelation function was stable above $D_{\max }$ values of $170 \AA$, and the optimal Total Score from GNOM was at a $D_{\max }$ value of $175 \AA$. A set of three final $p(R)$ functions was derived by setting the probability at $D_{\max }$ to be zero and setting $D_{\max }$ values at 170,175 , and $180 \AA$. These $p(R)$ functions were then used to determine the solution structure of activated NtrC (S160F, 3-Ala) eight independent times, for a total of 24 solutions, each time assuming sixfold symmetry. Each structure was determined on the LionXL cluster at Penn State, using the solution from GASBOR20 as an initial model for running GASBOR18 with the starting annealing temperature set at $1 \times 10^{-5}$ (Svergun et al. 2001). [GASBOR20 avoids Fourier transforms by fitting the real space $p(R)$ function; in contrast, GASBOR18 directly fits the reciprocal space scattering profile and usually, after a much longer time, arrives at a better solution. By applying the programs as described, we obtained the better fits with much reduced computer time.] The scattering curve calculated from one such solution is shown in Supplementary Figure S3A. Each of the eight independently derived solutions for a given $D_{\max }$ value were superimposed and averaged using DAMAVER (Volkov and Svergun 2003). As shown in Supplementary Table ST1, the average normalized spatial discrepancy value for each of the eight solutions for a given $p(R)$ function was within 2 standard deviations of the average for all comparisons. This is reflected by a close superpositioning of all eight solutions for a given $p(R)$ function (e.g., see Supplementary Fig. S3C for the set from $D_{\max }=175 \AA$ A). However, note in Supplementary Figure S3C that slices through the superimposed models reveal more dissimilarity for the DNA-binding domains than for the ATPase and receiver domains, perhaps reflecting conformational flexibility or an artifact of inappropriately imposing sixfold symmetry on the DNAbinding domains.

\section{Electron microscopy and image processing}

The oligomeric form of $\mathrm{Mg}^{2+} / \mathrm{BeF}_{3}{ }^{-}$-activated $\mathrm{NtrC}$ (S160F, 3-Ala) was isolated by size-exclusion chromatography using a 2.4-mL Superdex 200 gel filtration column (Amersham Pharmacia Biotech) in a SMART system in the presence of either ADP

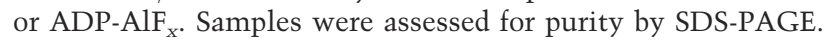
The sample was diluted to either $0.02 \mathrm{mg} / \mathrm{mL}$ (in the presence of $\mathrm{ADP}$ ) or $0.05 \mathrm{mg} / \mathrm{mL}$ (in the presence of $\mathrm{ADP}-\mathrm{AlF}_{\mathrm{x}}$ ) in a $\mathrm{Mg}^{2+}$ / $\mathrm{BeF}_{3}{ }^{-}$- and nucleotide-containing buffer $(200 \mathrm{mM} \mathrm{KCl}, 20 \mathrm{mM}$ Tris, $1 \mathrm{mM}$ nucleotide, $200 \mu \mathrm{M} \mathrm{BeCl}_{2}$, and $5 \mathrm{mM} \mathrm{NaF}, 5 \mathrm{mM}$ $\mathrm{MgCl}_{2}, 5 \%$ Trehalose at $\mathrm{pH} 8.2$ at $5^{\circ} \mathrm{C}$ ) and adsorbed onto a freshly glow-discharged carbon film support, mounted on 200mesh EM-grids. After $1 \mathrm{~min}$, the samples were stained with a $3 \%$ filtered solution of uranyl-acetate for $30 \mathrm{sec}$, blotted, and air-dried. For the ADP-bound sample, pairs of electron micrographs were collected at $0^{\circ}$ and $50^{\circ}$ in a Tecnai 12 electron microscope (FEI) operated at $120 \mathrm{kV}$, with a calibrated magnification of $49,687 \times$ and defocii ranging from 1.2 to $1.5 \mu \mathrm{m}$. For the ADP-AlF ${ }_{x}$-bound sample, the same conditions applied but only untilted $\left(0^{\circ}\right)$ views were collected.

Astigmatism- and drift-free micrographs were digitized using a Nikon SuperCoolscan ED8000 scanner at $2.56 \AA$ /pixel (at the specimen scale). For the ADP-bound NtrC sample, particles and their tilted counterparts were manually picked and extracted from the micrographs using WEB (Frank et al. 1996). All further processing was done using SPIDER (Frank et al. 1996). A total of 10,796 particles (5398 tilted and 5398 untilted) were boxed in $125 \times 125$ pixel windows. No CTF correction was applied, but the particles were filtered using a $24 \AA ̊$ Fermi low-pass filter to remove all information beyond the first CTF zero. Particles were normalized using histogram matching from a particle-free 
area (background) of the micrograph and centered using an iterative procedure that shifts the particle back to the center based on its rotational average. Twenty-four class averages representing single molecular views were obtained after three to four cycles of multivariate statistical analysis and hierarchical ascendant classification using Ward's criterion (Ward 1982) in SPIDER followed by 2D alignment. A reference-free, initial model of the oligomeric state of the protein in the presence of ADP was obtained using the random-conical tilt method (RTC) (Radermacher et al. 1987). Eight classes, including between 400 and 600 particles each, were selected based on their good signalto-noise ratio to generate eight corresponding class volumes, using the alignment parameters from the untilted particles and the estimated tilt angle to define the relative Euler angle of the tilted particles. The translational parameters of the tilted particles were refined by cross-correlation between each tilted particle and the corresponding reprojection of the volume in its direction (Penczek et al. 1992). A 3D orientation search including a rotation and cross-correlation steps was performed for all possible pairwise comparisons of the eight volumes. After each merger, a new extensive search was necessary in order to find the next best possible combination for merging. Visual inspection was used to validate the results or stop merging when it resulted in a loss of detail in the structure. Eight class volumes were ultimately merged into a final volume that included 2538 particles and showed well-defined features that match the initial class averages.

The merged, RTC volume was refined by projection-matching (Penczek et al. 1994), using the combined tilted and untilted data sets. Between refinement steps, the newly generated $3 \mathrm{D}$ model was filtered using a Fermi low-pass filter before being used as a new reference. Only three iterations were required to obtain a stable solution, with the final angular step set to $10^{\circ}$. The final reconstruction was computed using the best 7000 particles based on cross-correlation coefficient. This data set, used for the reconstruction in Figure 2A, shows a good angular distribution (Supplementary Fig. S4A, left) and led to a resolution of $25 \AA$ (Supplementary Fig. S4A, center).

$\mathrm{ADP}-\mathrm{AlF}_{\mathrm{x}}$ frequently used as a transition state analog for ATP hydrolysis, is known to stabilize the ring form of PspF and its complex with $\sigma^{54}$ (Chaney et al. 2001), and to alter the protein/DNA footprint of an activated form of DctD (Wang et al. 2003). Therefore a new full data set was obtained for activated NtrC (S160F, 3-Ala) in the presence of ADP-AlF ${ }_{x}$. EMAN (Ludtke et al. 1999) was used to semiautomatically pick 4670 particles. The particle locations (coordinates) were transferred to SPIDER using a homemade perl script. The 3D model for the ADP-bound state was filtered at $50 \AA$ and used as an initial reference for projection-matching. Only three rounds were necessary in order to obtain a stable solution, which included 3500 best particles selected based on their cross-correlation coefficient. In this case the "top-end" views were observed more frequently (Supplementary Fig. S4B, left), and the final reconstruction had a resolution of $28 \AA$ (Supplementary Fig. S4B, center). The final ADP- and ADP-AlF - -bound models were filtered to the same resolution and rendered with the same 4.6- $\sigma$ threshold (representing $\sim 100 \%$ of the expected molecular mass of $306 \mathrm{kDa}$, but see later) to allow for a better comparison.

In order to test the presence of rotational symmetry, the ADPand $\mathrm{ADP}-\mathrm{AlF}_{x}$ reconstructions were filtered to $32 \AA$, and their histogram normalized. A 3D cross-correlation coefficient (CCC) was then calculated between each reconstruction and its rotated versions in order to test for $N$-fold symmetry $(N=1-9)$. The cross-correlation process was carried out using two different $3 \mathrm{D}$ mask sizes, the first large enough to contain the whole 3D model, the second restricted to the ATPase central and DNAbinding domains; simulated sixfold or threefold symmetric data were used as a comparison (Supplementary Fig. S5). We found that the ADP-bound NtrC ring had good sixfold symmetry, no matter which mask was used. However, while the larger mask still showed sixfold symmetry, only threefold and twofold were present for the $\mathrm{ADP}-\mathrm{AlF}_{x}$ reconstruction when using the smaller mask. This result illustrates the fact that when the DNA-binding domains become ordered for this nucleotide state, they do not conform to the sixfold symmetry of the rest of the oligomer, but are present as three head-to-head dimers. Thus, in order to compare the EM density maps (Fig. 2C), sixfold and threefold symmetry were imposed on the ADP- and ADP$\mathrm{AlF}_{\mathrm{x}}$-bound states, respectively.

\section{Docking of the high-resolution structures}

A model of the NtrC ATPase ring was built based on the ATPase crystal structure of NtrC1 (PDB code 1NY6). We first generated an NtrC ATPase monomer model using the threading function of the DeepView program (Guex and Peitsch 1997) on the NtrC1 ATPase structure. An NtrC ATPase hexamer was then created by superimposing the monomer on the hexameric ring of NSF D2 (PDB code 1D2N). This model was then superimposed on the SAXS/WAXS structure using SUPCOMB13 (Kozin and Svergun 2001). Next, the NMR structures of the $\mathrm{NtrC} \mathrm{BeF}_{3}{ }^{-}$-bound receiver domain (PDB entry $1 \mathrm{KRW}$ ) and 3-Ala DNA-binding domain (PDB entry 1NTC) were positioned in the remaining density by hand. In doing so, helix 4 of the receiver domain was maintained near helix 1 of the ATPase domain as required by NMR (Hastings et al. 2003) and Fe-BABE cleavage studies (Lee 2000; Lee et al. 2000; Kustu, pers. comm.). We thus kept a distance of $12 \pm 3 \AA$ between the sites of tethering and their accompanying cleavages while optimizing surface charge interactions and the fit between the rest of the receiver domain and the solution model, revealing two dramatically different orientations (Supplementary Fig. S6A,B). Both dockings involve placing a negatively charged helix 4 near the positively charged C-terminal half of helix $\alpha \mathrm{N}$. But, only the orientation shown in Supplementary Figure S6A could be finetuned to involve numerous direct contacts between the side chains of the receiver domain that are surface-exposed in the phosphorylated state and the side chains of residues of helix $\alpha \mathrm{N}$ that generate phenotypic changes when replaced by cysteine (Supplementary Fig. S6C-F). Specifically, the acid moiety of D88 is shown interacting with either of the $\varepsilon$ amino groups of R152 or R156, the hydrophobic side chain of L87 contacts $C_{\beta}$ and $\mathrm{C}_{\gamma}$ of the R152 side chain, and the ring of F151, and the side chain of V91 contacts $C_{\beta}$ and $C_{\gamma}$ of D149. These postulated contacts seem reasonable and consistent with at least some of the reported phenotypes. For example, removing the positive charges in substitutions R152C and R156C could disrupt interactions with the acid moiety of D88, reducing the ability of the receiver domain to facilitate ring assembly and thus causing activation to fail. Substitution D149C causes partial constitutive activation-perhaps by improving interaction with the hydrophobic side chain of residue V91. Imposing sixfold symmetry in the SAXS/WAXS structure resulted in a continuous rim of density for the twofold symmetric DNA-binding domain. Three twofold-symmetric dimers were placed in the density in an arbitrary threefold symmetric way that optimized close proximity of the most $\mathrm{N}$-terminal region of each of the DNA-binding domains and the C-terminal portion of each ATPase domain. Coordinates for the SAXS/WAXS models shown in Supplementary Figure S3C and for the pseudoatomic model of the subdomains are available online as Supplemental Material (file NtrC.txt). In 
order to verify the consistency of this putative model with the EM data, it was interpolated at $320 \times 320 \times 320$ pixels to match the "absolute" scale of the PDB file and rendered using a threshold corresponding to $4.6 \sigma$. The electron density map was rescaled in SPIDER to the PDB coordinates (ribbons representation: receiver and ATPase domain are in alternative red/yellow; DNA-binding domains are in cyan/blue). The UCSF Chimera software (Pettersen et al. 2004) was used for display and manual fitting. The position of the six receiver domains along the $Z$-axis had to be slightly adjusted to best fit the EM density map. The

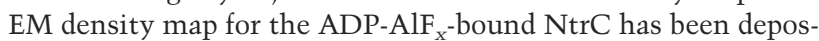
ited in the Electron Microscopy Database (EMDB) with accession code EMD-1218.

\section{Comparison of the SAXS and EM models}

Obtaining independent 3D EM reconstructions that correlate very well with the SAXS/WAXS model strongly supports the common structure and the full-length model docked into it. In addition, the structural methods are complementary. The SAXS/WAXS approach is a solution technique that provides data for macromolecules in their "native" states but requires relatively high concentrations of protein $(\geq 1 \mathrm{mg} / \mathrm{mL})$, can suffer from radiation damage, depends on having monodisperse aggregate-free solutions, and may require some a priori knowledge such as stoichiometry. In part, we could justifiably impose sixfold symmetry in our models based on the complementary information provided by the EM images, which uniformly and without imposing any symmetry showed hexamers. The assumption clearly failed when applied to the DNA-binding domains, both because of their apparent disorder in the ADPbound state and their $3 \times$ twofold symmetry when ordered in the ADP-AlF - -bound state. By virtue of the modeling process, the residues of the DNA-binding domains were included in modeling of the SAXS/WAXS data. All residues were positioned by a simulated annealing process that took into account a priori information about $\mathrm{C}_{\alpha}-\mathrm{C}_{\alpha}$ distance and local neighbors typically found in globular proteins (Svergun et al. 2001), much as typical bond lengths and bond angles are used to constrain models in refinement of crystallography models. The resulting model appears to have reproducibly yielded reasonable solutions, even though conformational variability of the DNA-binding domains in the ADP state resulted in the averaging out of this region in the EM reconstruction. It is a documented fact that flexible regions will disappear during the process of averaging of EM data, while SAXS, by nature, will give significant contributions for these disordered regions.

\section{Acknowledgments}

We thank Dmitri Svegun and Maxim Petoukhov for advice and modifying GASBOR to accommodate larger proteins, Sydney Kustu for supplying unpublished observations, and her and David Wemmer for making useful comments on the manuscript. We also thank Michael Cruse, Marian Peris, and Slaton Lipscomb for help with chromatography, biochemistry, and computer support, respectively. This work was funded by a Genomes to Life grant from the Department of Energy to E.N. and B.T.N. E.N. is a Howard Hughes Medical Institute Investigator. Use of the Advanced Photon Source was supported by the U.S. Department of Energy, Basic Energy Sciences, Office of Science, under contract no. W-31-109-ENG-38. BioCAT is a National Institutes of Health-supported Research Center, grant no. RR08630.

\section{References}

Bateman, A., Coin, L., Durbin, R., Finn, R.D., Hollich, V., Griffiths-Jones, S., Khanna, A., Marshall, M., Moxon, S., Sonnhammer, E.L., et al. 2004. The Pfam protein families database. Nucleic Acids Res. 32: D138-D141.

Chaney, M., Grande, R., Wigneshweraraj, S.R., Cannon, W., Casaz, P., Gallegos, M.T., Schumacher, J., Jones, S., Elderkin, S., Dago, A.E., et al. 2001. Binding of transcriptional activators to sigma 54 in the presence of the transition state analog ADP-aluminum fluoride: Insights into activator mechanochemical action. Genes \& Dev. 15: 2282-2294.

Demeler, B. 2005. UltraScan: A comprehensive data analysis software package for analytical ultracentrifugation experiments. In Modern analytical ultracentrifugation: Techniques and methods (eds. D.J. Scott et al.), pp. 210-230. Royal Society of Chemistry, Cambridge, UK.

Dixon, R., Eydmann, T., Henderson, N., and Austin, S. 1991. Substitutions at a single amino acid residue in the nitrogenregulated activator protein $\mathrm{NtrC}$ differentially influence its activity in response to phosphorylation. Mol. Microbiol. 5: 1657-1667.

Doucleff, M., Chen, B., Maris, A.E., Wemmer, D.E., Kondrashkina, E., and Nixon, B.T. 2005. Negative regulation of AAA+ ATPase assembly by two component receiver domains: A transcription activation mechanism that is conserved in mesophilic and extremely hyperthermophilic bacteria. J. Mol. Biol. 353: 242-255.

Drummond, M.H., Contreras, A., and Mitchenall, L.A. 1990. The function of isolated domains and chimaeric proteins constructed from the transcriptional activators NifA and NtrC of Klebsiella pneumoniae. Mol. Microbiol. 4: 29-37.

Fischetti, R.F., Rodi, D.J., Mirza, A., Irving, T.C., Kondrashkina, E., and Makowski, L. 2003. High-resolution wide-angle X-ray scattering of protein solutions: Effect of beam dose on protein integrity. J. Synchrotron Radiat. 10: 398-404.

Fischetti, R., Stepanov, S., Rosenbaum, G., Barrea, R., Black, E., Gore, D., Heurich, R., Kondrashkina, E., Kropf, A.J., Wang, S., et al. 2004. The BioCAT undulator beamline 18ID: A facility for biological non-crystalline diffraction and X-ray absorption spectroscopy at the Advanced Photon Source. J. Synchrotron Radiat. 11: 399-405.

Frank, J., Radermacher, M., Penczk, P., Zhu, J., Li, Y., Ladjadj, M., and Leith, A. 1996. SPIDER and WEB: Processing and visualization of images in $3 \mathrm{D}$ electron microscopy and related fields. J. Struct. Biol. 116: 190-199.

Guex, N. and Peitsch, M.C. 1997. SWISS-MODEL and the Swiss-Pdb Viewer: An environment for comparative protein modeling. Electrophoresis 18: 2714-2723.

Hansen, J.C., Lebowitz, J., and Demeler, B. 1994. Analytical ultracentrifugation of complex macromolecular systems. Biochemistry 33: 13155-13163.

Hastings, C.A., Lee, S.Y., Cho, H.S., Yan, D., Kustu, S., and Wemmer, D.E. 2003. High resolution solution structure of the beryllofluoride-activated $\mathrm{NtrC}$ receiver domain. Biochemistry 42: 9081-9090.

Hirschman, J., Wong, P.-K., Keener, J., and Kustu, S. 1985. Products of nitrogen regulatory genes $n \operatorname{tr} A$ and $n \operatorname{tr} C$ of enteric bacteria activate $g \ln A$ transcription in vitro: Evidence that the $n \operatorname{tr} A$ product is a $\sigma$ factor. Proc. Natl. Acad. Sci. 82: 7525-7529.

Kern, D., Volkman, B.F., Luginbuhl, P., Nohaile, M.J., Kustu, S., and Wemmer, D.E. 1999. Structure of a transiently phosphorylated switch in bacterial signal transduction. Nature 402: 894-898.

Klose, K.E., Weiss, D.S., and Kustu, S. 1993. Glutamate at the 
site of phosphorylation of nitrogen-regulatory protein NTRC mimics aspartyl-phosphate and activates the protein. J. Mol. Biol. 232: 67-78.

Koch, M.H. 2005. X-ray scattering of non-crystalline biological systems using synchrotron radiation. Chem. Soc. Rev. 35: 123-133.

Konarev, P.V., Volkov, V.V., Sokolova, A.V., Koch, M.H.J., and Svergun, D.I. 2003. PRIMUS: A Windows PC-based system for small-angle scattering data analysis. J. Appl. Crystallogr. 36: $1277-1282$.

Kozin, M.B. and Svergun, D.I. 2001. Automated matching of high- and low-resolution structural models. J. Appl. Crystallogr. 34: 33-41.

Lee, J.K. 2000. "Phosphorylation-induced interdomain communication in the response regulator NtrC of Salmonella typhimurium." Ph.D. thesis, University of California-Berkeley, Berkeley, CA.

Lee, J.K., Owens, J.T., Hwang, I., Meares, C., and Kustu, S. 2000. Phosphorylation-induced signal propagation in the response regulator NtrC. J. Bacteriol. 182: 5188-5195.

Lee, S.Y., DeLaTorre, A., Yan, D., Kustu, S., Nixon, B.T., and Wemmer, D.E. 2003. Regulation of the transcriptional activator NtrC1: Structural studies of the regulatory and AAA+ ATPase domains. Genes \& Dev. 17: 2552-2563.

Lilja, A.E., Jennsen, J.R., and Kahn, J.D. 2004. Geometric and dynamic requirements for DNA looping, wrapping and unwrapping in the activation of E. coli $g \ln A p 2$ transcription by NtrC. J. Mol. Biol. 342: 467-478.

Ludtke, S.J., Baldwin, P.R., and Chiu, W. 1999. EMAN: Semiautomated software for high-resolution single-particle reconstructions. J. Struct. Biol. 128: 82-97.

Merrick, M.J. and Edwards, R.A. 1995. Nitrogen control in bacteria. Microbiol. Rev. 59: 604-622.

Meyer, M., Park, S., Zeringue, L., Staley, M., McKinstry, M., Kaufman, R.I., Zhang, H., Yan, D., Yennawar, N., Yennawar, H., et al. 2001. A dimeric two-component receiver domain inhibits the $\sigma^{54}$-dependent ATPase in DctD. FASEB J. 15: 1326-1328.

Moore, P.B. 1980. Small-angle scattering, information content and error analysis. J. Appl. Crystallogr. 13: 168-175.

Neuwald, A.F., Aravind, L., Spounge, J.L., and Koonin, E.V. 1999. AAA+: A class of chaperonin-like ATPases associated with the assembly, operation, and disassembly of protein complexes. Genome Res. 9: 27-43.

North, A.K. and Kustu, S. 1997. Mutant forms of the enhancerbinding protein NtrC can activate transcription from solution. J. Mol. Biol. 267: 17-36.

Osuna, J., Soberon, X., and Morett, E. 1997. A proposed architecture for the central domain of the bacterial enhancerbinding proteins based on secondary structure prediction and fold recognition. Protein Sci. 6: 543-555.

Park, S., Meyer, M., Jones, A.D., Yennawar, H., Yennawar, N., and Nixon, B.T. 2002. Two-component signaling in the $\mathrm{AAA}+$ ATPase DctD: Binding $\mathrm{Mg}^{2+}$ and $\mathrm{BeF}^{-}$selects between alternative dimeric states of the receiver domain. FASEB J. 16: 1964-1966.

Pelton, J.G., Kustu, S., and Wemmer, D.E. 1999. Solution structure of the DNA-binding domain of NtrC with three alanine substitutions. J. Mol. Biol. 292: 1095-1110.

Penczek, P., Radermacher, M., and Frank, J. 1992. Three-dimensional reconstruction of single particles embedded in ice. Ultramicroscopy 40: 33-53.

Penczek, P., Grassucci, R.A., and Frank, J. 1994. The ribosome at improved resolution: New techniques for merging and orientation refinement in $3 \mathrm{D}$ cryoelectron microscopy of biological particles. Ultramicroscopy 53: 251-270.
Pettersen, E.F., Goddard, T.D., Huang, C.C., Couch, G.S., Greenblatt, D.M., Meng, E.C., and Ferrin, T.E. 2004. UCSF Chimera-A visualization system for exploratory research and analysis. J. Comput. Chem. 25: 1605-1612.

Phillips, W.C., Stewart, A., Stanton, M., Naday, I., and Ingersoll, C. 2002. High-sensitivitiy CCD-based X-ray detector. J. Synchrotron Radiat. 9: 36-43.

Popham, D.L., Szeto, D., Keener, J., and Kustu, S. 1989. Function of a bacterial enhancer activator protein that binds to transcriptional activators. Science 243: 629-635.

Porter, S.C., North, A.K., and Kustu, S. 1995. Mechanism of transcriptional activation by NtrC. In Two component signal transduction (eds. J.A. Hoch and T.J. Silhavy), pp. 147-158. American Society for Microbiology, Washington, DC.

Radermacher, M., Wagenknecht, T., Verschoor, A., and Frank, J. 1987. Three-dimensional reconstruction from a single-exposure, random conical tilt series applied to the 50S ribosomal subunit of Escherichia coli. J. Microsc. 146: 113-136.

Rappas, M., Schumacher, J., Beuron, F., Niwa, H., Bordes, P., Wigneshweraraj, S.R., Keetch, C.A., Robinson, C.V., Buck, M., and Zhang, X. 2005. Structural insights into the activity of enhancer-binding proteins. Science 307: 19721975.

Reitzer, L. 2003. Nitrogen assimilation and global regulation in Escherichia coli. Annu. Rev. Microbiol. 57: 155-176.

Rippe, K., Mucke, N., and Schulz, A. 1998. Association states of the transcription activator protein NtrC from E. coli determined by analytical ultracentrifugation. J. Mol. Biol. 278: 915-933.

Rombel, I., North, A.K., Hwang, I., Wyman, C., and Kustu, S. 1998. The bacterial enhancer-binding protein NtrC as a molecular machine. Cold Spring Harb. Symp. Quant. Biol. 63: 157-166.

Sallai, L. and Tucker, P.A. 2005. Crystal structure of the central and C-terminal domain of the $\sigma^{54}$-activator ZraR. J. Struct. Biol. 151: 160-170.

Sasse-Dwight, S. and Gralla, J.D. 1988. Probing the E. coli glnALG upstream activation mechanism in vivo. Proc. Natl. Acad. Sci. 85: 8934-8938.

Svergun, D.I. 1992. Determination of the regularization parameter in indirect-transform methods using perceptual criteria. J. Appl. Crystallogr. 25: 495-503.

Svergun, D.I., Petoukhov, M.V., and Koch, M.H.J. 2001. Determination of domain structure of proteins from X-ray solution scattering. Biophys. J. 80: 2946-2953.

Vachette, P., Koch, M.H., and Svergun, D.I. 2003. Looking behind the beamstop: X-ray solution scattering studies of structure and conformational changes of biological macromolecules. Methods Enzymol. 374: 584-615.

Volkman, B.F., Nohaile, M.J., Amy, N.K., Kustu, S., and Wemmer, D.E. 1995. Three dimensional solution structure of the $\mathrm{N}$-terminal receiver domain of NtrC. Biochemistry 34: 1413-1424.

Volkov, V.V. and Svergun, D.I. 2003. Uniqueness of ab initio shape determination in small-angle scattering. J. Appl. Crystallogr. 36: 860-864.

Wang, Y.-K., Park, S., Nixon, B.T., and Hoover, T.R. 2003. Nucleotide-dependent conformational changes in the $\sigma^{54}$ dependent activator DctD. J. Bacteriol. 185: 6215-6219.

Ward, J.H. 1982. Hierarchical grouping to optimize an objective function. Am. Stat. Assoc. 58: 236-244.

Weiss, D.S., Batut, J., Klose, K.E., Keener, J., and Kustu, S. 1991. The phosphorylated form of the enhancer binding protein NtrC has an ATPase activity that is essential for activation of transcription. Cell 67: 155-168. 
Weiss, D.S., Klose, K.E., Hoover, T.R., North, A.K., Porter, S.C., Wedel, A.B., and Kustu, S. 1992a. Prokaryotic transcriptional enhancers. In Transcriptional regulation (eds. S. McKnight and K. Yammamoto), pp. 667-694. Cold Spring Harbor Laboratory Press, Cold Spring Harbor, NY.

Weiss, V., Claverie-Martin, F., and Magasanik, B. 1992b. Phosphorylation of nitrogen regulator I of Escherichia coli induces strong cooperative binding to DNA essential for activation of transcription. Proc. Natl. Acad. Sci. 89: 5088-5092.

Yan, D. and Kustu, S. 1999. "Switch I" mutant forms of the bacterial enhancer-binding protein NtrC that perturb the response to DNA. Proc. Natl. Acad. Sci. 96: 13142-13146.

Yan, D., Cho, H.S., Hastings, C.A., Igo, M.M., Lee, S.-Y., Pelton, J.G., Stewart, V., Wemmer, D.E., and Kustu, S. 1999. Beryllofluoride mimics phosphorylation of NtrC and other bacterial response regulators. Proc. Nat1. Acad. Sci. 96: 1478914794.

Zimmer, D.P., Soupene, E., Lee, H.L., Wendisch, V.F., Khodursky, A.B., Peter, B.J., Bender, R.A., and Kustu, S. 2000. Nitrogen regulatory protein C-controlled genes of Escherichia coli: Scavenging as a defense against nitrogen limitation. Proc. Natl. Acad. Sci. 97: 14674-14679. 


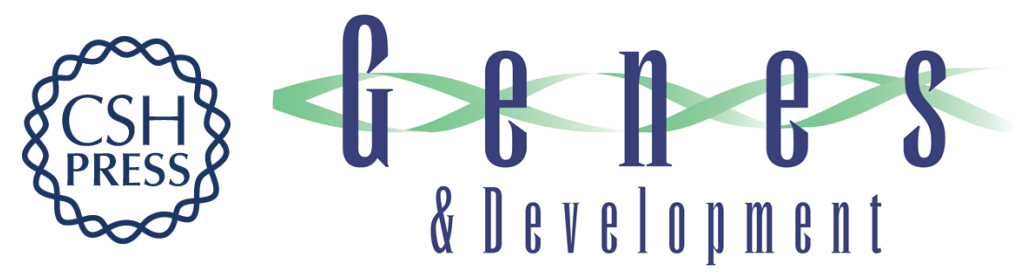

\section{The structural basis for regulated assembly and function of the transcriptional activator NtrC}

Sacha De Carlo, Baoyu Chen, Timothy R. Hoover, et al.

Genes Dev. 2006, 20:

Access the most recent version at doi:10.1101/gad.1418306

\section{Supplemental http://genesdev.cshlp.org/content/suppl/2006/05/16/20.11.1485.DC1 Material}

References This article cites 52 articles, 14 of which can be accessed free at: http://genesdev.cshlp.org/content/20/11/1485.full.html\#ref-list-1

\section{License}

Email Alerting

Receive free email alerts when new articles cite this article - sign up in the box at the top Service 